\title{
AN ANALYSIS OF NEW FINITE ELEMENT SPACES FOR MAXWELL'S EQUATIONS
}

\section{JI HYUN KIM}

Department of Mathematics

Hannam University

133 Ojeong-dong

Daedeok-gu, Daejeon 306-791

Republic of Korea

e-mail:1m97jh@kaist.ac.kr

\begin{abstract}
In this paper, we introduce four different finite element spaces on hexahedron for Maxwell's equations. We also describe interpolation operators that map from suitable subspaces $H^{1}(\Omega), H(\operatorname{curl}, \Omega), H(\operatorname{div}, \Omega)$ and the space $L^{2}(\Omega)$ into the appropriate finite element spaces. For the error analysis, we prove that the spaces and interpolation operators are linked by the discrete de Rham diagram.
\end{abstract}

\section{Introduction}

In performing the analysis of finite element methods for Maxwell's equations, it is necessary to present four different finite element spaces. The most obvious requirement is the curl conforming edge elements suitable for discretizing the basic energy space for electromagnetics. The most common example is Nedelec elements given in [7]. Recently, we 2010 Mathematics Subject Classification: 65N30, 65N12.

Keywords and phrases: $H^{1}$ conforming element, edge element, divergence conforming element, $L^{2}$ conforming element, de Rham diagram.

Received June 11, 2018

(ㄷ) 2018 Scientific Advances Publishers 
have introduced a new family of curl conforming finite elements on parallelepiped grids in [5], with fewer degrees of freedom than the existing ones. To analyze edge elements, we also need to present the divergence conforming elements and scalar finite elements. Well-known examples for the divergence conforming element spaces are the RaviartThomas-Nedelec (RTN) space and the Brezzi-Douglas-Duran-Fortin (BDDF) space [4]. We have introduced a new family of divergence conforming elements, lying between RTN and BDDF spaces [5]. Although BDDF space has less degrees of freedom than our new element space, there is no finite element corresponding to the curl conforming finite element space. For the scalar finite elements, we also have presented a new elements in [6]. For completeness, we shall present a related new finite element family in $L^{2}(\Omega)$. We will describe interpolation operators $\pi_{h}, \mathbf{r}_{h}, \omega_{h}$, and $P_{0, h}$ that map from suitable subspaces $U \subset H^{1}(\Omega), \mathbf{V} \subset H(\operatorname{curl}, \Omega), \mathbf{W} \subset H(\operatorname{div}, \Omega)$ and the space $L^{2}(\Omega)$ into the appropriate finite element spaces and prove the interrelationship of the spaces linked by the discrete de Rham diagram.

\section{Four Finite Element Spaces}

In this section, we will describe four different finite element spaces on hexahedra with edges parallel to the coordinate axes. First, we generate a regular finite element mesh $\tau_{h}=\{K\}, h>0$ covering the domain $\Omega$ such that

(1) $\bar{\Omega}=\bigcup_{K \in \tau_{h}} \bar{K}$, where $\bar{\Omega}$ denotes the closure of $\Omega$;

(2) for each $K \in \tau_{h}, K$ is an open set with positive volume;

(3) if $K_{1}$ and $K_{2}$ are distinct elements in $\tau_{h}$, then $K_{1} \bigcap K_{2}=\varnothing$;

(4) each $K \in \tau_{h}$ is a Lipschitz domain. 
For each element $K$, we define the parameters $h_{K}$ and $\rho_{K}$ such that

$$
\begin{aligned}
& h_{K}=\text { diameter of the smallest sphere containing } \bar{K}, \\
& \rho_{K}=\text { diameter of the largest sphere contained in } \bar{K} \text {, }
\end{aligned}
$$

then $h=\max _{K \in \tau_{h}} h_{K}$ so that the index $h$ denote the maximum diameter of the elements $K \in \tau_{h}$. Any $K \in \tau_{h}$ can be obtained by mapping the reference element $\widehat{K}$, typically unit cube, using an affine map $F_{K}: \widehat{K} \rightarrow K$ such that $F_{K}(\widehat{K})=K$ and $F_{K}(\hat{\mathbf{x}})=B_{K} \hat{\mathbf{x}}+b_{K}$, where $B_{K}$ is an invertible diagonal matrix and $b_{K}$ is a vector. We shall be concerned with mapping between functions defined on the reference element $\widehat{K}$ and the target element $K$. For a simple scalar function $\hat{p}$ defined on $\widehat{K}$, we obtain a corresponding function $p$ on $K$ by

$$
p \circ F_{K}=\hat{p}
$$

where $\circ$ denotes composition of functions. And a simple calculation using the chain rule shows that

$$
(\nabla p) \circ F_{K}=B_{K}^{-T} \hat{\nabla} \hat{p}
$$

where $\hat{\nabla}$ is the gradient with respect to $\hat{\mathbf{x}}$. However, vector functions must be transformed in a more careful way to conserve their properties. For $\hat{\mathbf{v}} \in H(\operatorname{curl}, \widehat{K})$, we define $\mathbf{v}$ on $K$ by

$$
\mathbf{v} \circ F_{K}=B_{K}^{-T} \hat{\mathbf{v}}
$$

And if $\hat{\mathbf{w}} \in H(\operatorname{div}, \widehat{K})$, then we define $\mathbf{w}$ by

$$
\mathbf{w} \circ F_{K}=\frac{1}{\operatorname{det}\left(B_{K}\right)} B_{K} \hat{\mathbf{w}}
$$


Because of the simplicity of the mapping, although we define the elements on the reference domain $\widehat{K}$, the same definition can be used on a target element $K$ in the mesh [8]. In order to define finite elements on parallelepiped, we need the following polynomial spaces:

$Q_{\ell, m, n}(\widehat{K})=\{$ polynomials of maximum degree $\ell$ in $\hat{x}, m$ in $\hat{y}$ and $n$ in $\hat{z}$ \}.

\section{1. $H^{1}(\Omega)$ conforming finite element spaces}

We start by defining the scalar spaces suitable for discretizing the potential [6].

Definition 1. Let $k \geq 1$. On the reference element $\widehat{K}$, the gradient conforming element is defined as follows:

$$
\widehat{U}(\widehat{K})=Q_{k+1, k+1, k+1}^{*}(\widehat{K}),
$$

where $Q_{k+1, k+1, k+1}^{*}(\widehat{K})$ is the subspace of $Q_{k+1, k+1, k+1}(\widehat{K})$ except constant multiple of the term $\hat{x}^{k+1} \hat{y}^{k+1} \hat{z}^{\ell}, \hat{x}^{k+1} \hat{y}^{\ell} \hat{z}^{k+1}, \hat{x}^{\ell} \hat{y}^{k+1} \hat{z}^{k+1}$, and $\hat{x}^{k+1} \hat{y}^{k+1} \hat{z}^{k+1}$ for $\ell=0 \ldots k$.

Then we see the dimension of $\widehat{U}(\widehat{K})$ is $(k+2)^{3}-3(k+1)-1$.

Definition 2. For any $\hat{p} \in \widehat{U}(\widehat{K})$, we define the following degrees of freedom:

$$
\begin{gathered}
\hat{p}(\hat{\mathbf{a}}), \text { for the eight vertices } \hat{\mathbf{a}} \text { of } \widehat{K}, \\
\int_{\hat{e}} \hat{p} \hat{q} d \hat{s}, \text { for each edges } \hat{e} \text { of } \widehat{K}, \quad \hat{q} \in P_{k-1}(\hat{e}), \\
\int_{\hat{f}} \hat{p} \hat{q} d \hat{A}, \text { for each faces } \hat{f} \text { of } \hat{K}, \quad \hat{q} \in Q_{k-1, k-1}^{*}(\hat{f}), \\
\int_{\widehat{K}} \hat{p} \hat{q} d \hat{A}, \quad \hat{q} \in Q_{k-1, k-1, k-1}^{*}(\widehat{K}),
\end{gathered}
$$


where $Q_{k-1, k-1}^{*}(\hat{f})$ is the subspace of $Q_{k-1, k-1}(\hat{f})$ except constant multiple of the term $\hat{x}^{k-1} \hat{y}^{k-1}, \hat{y}^{k-1} \hat{z}^{k-1}, \hat{z}^{k-1} \hat{x}^{k-1}$, and $Q_{k-1, k-1, k-1}^{*}(\widehat{K})$ is the subspace of $Q_{k-1, k-1, k-1}(\widehat{K})$ except constant multiple of the term $\hat{x}^{k-1} \hat{y}^{k-1} \hat{z}^{\ell}, \hat{x}^{k-1} \hat{y}^{\ell} \hat{z}^{k-1}, \hat{x}^{\ell} \hat{y}^{k-1} \hat{z}^{k-1}$, and $\hat{x}^{k-1} \hat{y}^{k-1} \hat{z}^{k-1}$ for $\ell=0 \ldots k-2$.

Theorem 3. A scalar function $\hat{p} \in \widehat{U}(\widehat{K})$ is uniquely determined by the degrees of freedom (1)-(4). And the finite element space $\widehat{U}(\widehat{K})$ is conforming in $H^{1}(\Omega)$.

Proof. Since the number of degrees of freedom and the dimension of $\widehat{U}(\widehat{K})$ are both $k^{3}+6 k^{2}+9 k+4$, it suffices to show that if $\hat{p} \in \widehat{U}(\widehat{K})$ and all the degrees of freedom (1)-(4) of $\hat{p}$ vanish, then $\hat{p}=0$. First, we use the fact that the vertex degrees of freedom vanish on each edge $\hat{e}$ of $\hat{f}$. For example, on the edge $\hat{x}=\hat{y}=0$ we have

$$
\hat{p}=\hat{z}(1-\hat{z}) r
$$

for some $r \in P_{k-1}(\hat{e})$. Choosing $\hat{q}=r$ in the degrees of freedom (2) for this edge shows that $r=0$. Now using the fact that $\hat{p}=0$ on each edge $\hat{e}$ of $\hat{f}$, which we assume to be the face $\hat{z}=0$, we have

$$
\hat{p}=\hat{x}(1-\hat{x}) \hat{y}(1-\hat{y}) r,
$$

for some $r \in Q_{k-1, k-1}^{*}(\hat{f})$. Choosing $\hat{q}=r$ in the degrees of freedom (3) shows that $r=0$ and hence $\hat{p}=0$ on $\hat{f}$. By the same reason, we see that $\hat{p}=0$ on all faces. This proves the conformity in $H^{1}(\Omega)$ space. And we have

$$
\hat{p}=\hat{x}(1-\hat{x}) \hat{y}(1-\hat{y}) \hat{z}(1-\hat{z}) r
$$

for some $r \in Q_{k-1, k-1, k-1}^{*}(\widehat{K})$. Choosing $\hat{q}=r$ in the degrees of freedom (4) proves $r=0$ and hence $\hat{p}=0$. 
Remark 4. Our element has smaller number of degrees of freedom than the well known $H^{1}(\Omega)$ conforming finite elements on parallelepiped.

The finite element space on a general element $K$ can be obtained by using the diagonal affine map $F_{K}$ via

$$
U(K)=\left\{\hat{p} \circ F_{K}^{-1} \mid \hat{p} \in \widehat{U}(\widehat{K})\right\}
$$

in the usual way. Then we have the following space:

$$
U_{h}=\left\{p \in H^{1}(\Omega)|p|_{K} \in U(K) \text { for all } K \in \tau_{h}\right\} .
$$

Using the degrees of freedom (1)-(4) transformed on $K$, we can define an interpolation operator

$$
\pi_{K}: H^{k+1}(K) \rightarrow U(K)
$$

by requiring the degrees of freedom of $\pi_{K} p-p$ vanish. Then the global interpolation operator $\pi_{h}$ is defined element-wise by

$$
\left.\left(\pi_{h} p\right)\right|_{K}=\pi_{K}\left(\left.p\right|_{K}\right)
$$

for all $K \in \tau_{h}$.

\subsection{The curl conforming finite element spaces}

We now present the edge elements which is used to discretize the electric field [6].

Definition 5. Let $k \geq 1$. On the reference element $\widehat{K}$, the curl conforming element space $\widehat{\mathrm{V}}(\widehat{K})$ is defined by the subspace of $Q_{k, k+1, k+1}(\widehat{K}) \times Q_{k+1, k, k+1}(\widehat{K}) \times Q_{k+1, k+1, k}(\widehat{K})$, where the elements in the set $\left\{\hat{\alpha}_{i, j}\right\}_{j=1,2}$ are replaced by the elements $\hat{\beta}_{i}$, and the three elements $\hat{\gamma}_{i}$ are replaced by the single element $\hat{\delta}$ for $i=1,2,3$ as follows: 


$$
\begin{aligned}
& \hat{\alpha}_{11}=\left\{\left(0, \hat{x}^{k+1} \hat{y}^{k} \hat{z}^{\ell}, 0\right)\right\}_{\ell=0}^{k} \Rightarrow \hat{\beta}_{1}=\left\{\left(\hat{x}^{k} \hat{y}^{k+1} \hat{z}^{\ell}, \hat{x}^{k+1} \hat{y}^{k} \hat{z}^{\ell}, 0\right)\right\}_{\ell=0}^{k}, \\
& \hat{\alpha}_{12}=\left\{\left(\hat{x}^{k} \hat{y}^{k+1} \hat{z}^{\ell}, 0,0\right)\right\}_{\ell=0}^{k} \\
& \hat{\alpha}_{21}=\left\{\left(0,0, \hat{x}^{\ell} \hat{y}^{k+1} \hat{z}^{k}\right)\right\}_{\ell=0}^{k} \Rightarrow \hat{\beta}_{2}=\left\{\left(0, \hat{x}^{\ell} \hat{y}^{k} \hat{z}^{k+1}, \hat{x}^{\ell} \hat{y}^{k+1} \hat{z}^{k}\right)\right\}_{\ell=0}^{k}, \\
& \hat{\alpha}_{22}=\left\{\left(0, \hat{x}^{\ell} \hat{y}^{k} \hat{z}^{k+1}, 0\right)\right\}_{\ell=0}^{k} \\
& \hat{\alpha}_{31}=\left\{\left(0,0, \hat{x}^{k+1} \hat{y}^{\ell} \hat{z}^{k}\right)\right\}_{\ell=0}^{k} \Rightarrow \hat{\beta}_{3}=\left\{\left(\hat{x}^{k} \hat{y}^{\ell} \hat{z}^{k+1}, 0, \hat{x}^{k+1} \hat{y}^{\ell} \hat{z}^{k}\right)\right\}_{\ell=0}^{k}, \\
& \hat{\alpha}_{32}=\left\{\left(\hat{x}^{k} \hat{y}^{\ell} \hat{z}^{k+1}, 0,0\right)\right\}_{\ell=0}^{k} \\
& \hat{\gamma}_{1}=\left\{\left(\hat{x}^{k} \hat{y}^{k+1} \hat{z}^{k+1}, 0,0\right)\right\} \\
& \hat{\gamma}_{2}=\left\{\left(0, \hat{x}^{k+1} \hat{y}^{k} \hat{z}^{k+1}, 0\right)\right\} \Rightarrow \hat{\delta}=\left\{\left(\hat{x}^{k} \hat{y}^{k+1} \hat{z}^{k+1}, \hat{x}^{k+1} \hat{y}^{k} \hat{z}^{k+1}, \hat{x}^{k+1} \hat{y}^{k+1} \hat{z}^{k}\right)\right\} . \\
& \hat{\gamma}_{3}=\left\{\left(0,0, \hat{x}^{k+1} \hat{y}^{k+1} \hat{z}^{k}\right)\right\}
\end{aligned}
$$

Then the dimension of $\widehat{\mathbf{V}}(\widehat{K})$ is $3(k+1)(k+2)^{2}-3(k+1)-2$.

To define the degrees of freedom, we need two auxiliary spaces. First, we define $\widehat{\Phi}_{k}^{\text {curl }}(\hat{x}, \hat{y})$ to be the subspace of $Q_{k-1, k}(\hat{x}, \hat{y}) \times Q_{k, k-1}(\hat{x}, \hat{y})$, where the two elements $\left(\hat{x}^{k-1}, \hat{y}^{k}, 0\right)$ and $\left(0, \hat{x}^{k} \hat{y}^{k-1}\right)$ are replaced by the single element $\left(\hat{x}^{k-1} \hat{y}^{k}, \hat{x}^{k} \hat{y}^{k-1}\right)$. To define the second space, we use a replacement rule similar to the Definition 5. We define $\widehat{\Psi}_{k}^{\text {curl }}(\widehat{K})$ to be the subspace of $Q_{k, k-1, k-1}(\widehat{K}) \times Q_{k-1, k, k-1}(\widehat{K}) \times Q_{k-1, k-1, k}(\widehat{K})$, where the elements $\left\{\hat{\phi}_{i j}\right\}_{j=1,2}$ are replaced by the elements $\hat{\psi}_{i}$ and the three elements $\hat{\xi}_{i}$ are replaced by the single element $\hat{\zeta}$ for $i=1,2,3$ as follows:

$$
\begin{aligned}
& \hat{\phi}_{11}=\left\{\left(0, \hat{x}^{k-1} \hat{y}^{k} \hat{z}^{\ell}, 0\right)\right\}_{\ell=0}^{k-2} \\
& \hat{\phi}_{12}=\left\{\left(\hat{x}^{k} \hat{y}^{k-1} \hat{z}^{\ell}, 0,0\right)\right\}_{\ell=0}^{k-2}
\end{aligned} \Rightarrow \hat{\psi}_{1}=\left\{\left(\hat{x}^{k} \hat{y}^{k-1} \hat{z}^{\ell}, \hat{x}^{k-1} \hat{y}^{k} \hat{z}^{\ell}, 0\right)\right\}_{\ell=0}^{k-2},
$$




$$
\begin{aligned}
& \hat{\phi}_{21}=\left\{\left(0,0, \hat{x}^{\ell} \hat{y}^{k-1} \hat{z}^{k}\right)\right\}_{\ell=0}^{k-2} \\
& \hat{\phi}_{22}=\left\{\left(0, \hat{x}^{\ell} \hat{y}^{k} \hat{z}^{k-1}, 0\right)\right\}_{\ell=0}^{k-2} \\
& \Rightarrow \hat{\psi}_{2}=\left\{\left(0, \hat{x}^{\ell} \hat{y}^{k} \hat{z}^{k+1}, \hat{x}^{\ell} \hat{y}^{k-1} \hat{z}^{k}\right)\right\}_{\ell=0}^{k-2}, \\
& \hat{\phi}_{31}=\left\{\left(0,0, \hat{x}^{k-1} \hat{y}^{\ell} \hat{z}^{k}\right)\right\}_{\ell=0}^{k-2} \\
& \Rightarrow \hat{\psi}_{3}=\left\{\left(\hat{x}^{k} \hat{y}^{\ell} \hat{z}^{k-1}, 0, \hat{x}^{k-1} \hat{y}^{\ell} \hat{z}^{k}\right)\right\}_{\ell=0}^{k-2}, \\
& \hat{\phi}_{32}=\left\{\left(\hat{x}^{k} \hat{y}^{\ell} \hat{z}^{k-1}, 0,0\right)\right\}_{\ell=0}^{k-2} \\
& \hat{\xi}_{1}=\left\{\left(\hat{x}^{k} \hat{y}^{k-1} \hat{z}^{k-1}, 0,0\right)\right\} \\
& \hat{\xi}_{2}=\left\{\left(0, \hat{x}^{k-1} \hat{y}^{k} \hat{z}^{k-1}, 0\right)\right\} \Rightarrow \hat{\zeta}=\left\{\left(\hat{x}^{k} \hat{y}^{k-1} \hat{z}^{k-1}, \hat{x}^{k-1} \hat{y}^{k} \hat{z}^{k-1}, \hat{x}^{k-1} \hat{y}^{k-1} \hat{z}^{k}\right)\right\} . \\
& \hat{\xi}_{3}=\left\{\left(0,0, \hat{x}^{k-1} \hat{y}^{k-1} \hat{z}^{k}\right)\right\}
\end{aligned}
$$

Definition 6. For any $\hat{\mathbf{v}} \in \widehat{\mathbf{V}}(\widehat{K})$, the degrees of freedom are given on edge $\hat{e}$ with unit tangent $\hat{\mathbf{t}}$, on faces $\hat{f}$ with unit normal $\hat{\mathbf{n}}$ and in the interior of $\widehat{K}$ as follows:

$$
\begin{gathered}
\int_{\hat{e}} \hat{\mathbf{v}} \cdot \hat{\mathbf{t}} \hat{q} d \hat{s}, \text { for each edges } \hat{e} \text { of } \widehat{K}, \quad \hat{q} \in P_{k}(\hat{e}), \\
\int_{\hat{f}}(\hat{\mathbf{v}} \times \hat{\mathbf{n}}) \cdot \hat{\mathbf{q}} d \hat{A}, \quad \text { for each faces } \hat{f} \text { of } \widehat{K}, \quad \hat{\mathbf{q}} \in \widehat{\Phi}_{k}^{\operatorname{curl}}(\hat{f}), \\
\int_{\widehat{K}} \hat{\mathbf{v}} \cdot \hat{\mathbf{q}} d \hat{\mathbf{x}}, \quad \hat{\mathbf{q}} \in \widehat{\Psi}_{k}^{\operatorname{curl}}(\widehat{K}) .
\end{gathered}
$$

Theorem 7. A vector function $\hat{\mathbf{u}} \in \widehat{\mathbf{V}}(\widehat{K})$ is uniquely determined by the degrees of freedom (6)-(8). And the finite element space $\widehat{\mathbf{V}}(\widehat{K})$ is conforming in $H(\operatorname{curl}, \Omega)$.

Proof. Since the number of conditions and the dimension of $\widehat{\mathbf{V}}(\widehat{K})$ are both $3 k^{3}+15 k^{2}+21 k+7$, it suffices to show that if all the conditions are zero, then $\hat{\mathbf{u}}=0$. First, we consider the face $\hat{z}=0$. Then the tangential component of $\hat{\mathbf{u}}$ on this face is $\left(\hat{u}_{1}, \hat{u}_{2}\right) \in Q_{k, k+1}(\hat{x}, \hat{y}) \times$ 
$Q_{k+1, k}(\hat{x}, \hat{y})$. On each edge of this face, the tangential component is polynomial of degree $k$. From the degrees of freedom in (6), we see that $\hat{\mathbf{u}} \cdot \hat{\mathbf{t}}=0$ on each edge. This implies that on this face, we have

$$
\hat{u}_{1}=\hat{y}(1-\hat{y}) \hat{v}_{1}, \hat{u}_{2}=\hat{x}(1-\hat{x}) \hat{v}_{2}
$$

where $\left(\hat{v}_{1}, \hat{v}_{2}\right) \in \widehat{\Phi}_{k}^{\text {curl }}(\hat{x}, \hat{y})$. Then by choosing $\hat{q}_{1}=\hat{v}_{2}$ and $\hat{q}_{2}=-\hat{v}_{1}$ in the degrees of freedom (7), we see $\hat{v}_{1}=\hat{v}_{2}=0$. Hence $\hat{\mathbf{u}} \times \hat{\mathbf{n}}=0$ on this face. By the same reason, we see that $\hat{\mathbf{u}} \times \hat{\mathbf{n}}=0$ on all faces. This proves the conformity in $H$ (curl) space. And we have

$$
\begin{aligned}
& \hat{u}_{1}=\hat{y}(1-\hat{y}) \hat{z}(1-\hat{z}) \hat{w}_{1}, \\
& \hat{u}_{2}=\hat{x}(1-\hat{x}) \hat{z}(1-\hat{z}) \hat{w}_{2}, \\
& \hat{u}_{3}=\hat{x}(1-\hat{x}) \hat{y}(1-\hat{y}) \hat{w}_{3},
\end{aligned}
$$

where $\left(\hat{w}_{1}, \hat{w}_{2}, \hat{w}_{3}\right) \in \widehat{\Psi}_{k}^{\text {curl }}(\widehat{K})$. Choosing $\hat{\mathbf{q}}=\left(\hat{w}_{1}, \hat{w}_{2}, \hat{w}_{3}\right)$ in degrees of freedom (8), we know that $\hat{\mathbf{u}}=0$.

Remark 8. Our element space has $3 k+5$ fewer degrees of freedom than the well known Nedelec finite element space in each element. Hence it is more efficient.

For a generic element $K$, we define the finite element space on $K$ as

$$
\mathbf{V}(K)=\left\{\left(B_{K}^{T}\right)^{-1} \hat{\mathbf{v}} \circ F_{K}^{-1} \mid \hat{\mathbf{v}} \in \widehat{\mathbf{V}}(\widehat{K})\right\},
$$

in the usual way. Then we have the following space:

$$
\mathbf{V}_{h}=\left\{\mathbf{v} \in H(\operatorname{curl}, \Omega)|\mathbf{v}|_{K} \in \mathbf{V}(K) \text { for all } K \in \tau_{h}\right\} .
$$

Using the degrees of freedom (6)-(8) transformed on $K$, we can define the corresponding projection

$$
\mathbf{r}_{K}: \mathbf{H}^{k+1}(K) \rightarrow \mathbf{V}(K)
$$


for an arbitrary element $K$. Then the global projection operator $\mathbf{r}_{h}$ is defined piecewise by

$$
\left.\left(\mathbf{r}_{h} \mathbf{v}\right)\right|_{K}=\mathbf{r}_{K}\left(\left.\mathbf{v}\right|_{K}\right)
$$

for all $K \in \tau_{h}$.

\subsection{The divergence conforming finite element spaces}

In this subsection, we introduce divergence conforming finite element spaces which will be used to discretize the magnetic induction [5].

Definition 9. Let $k \geq 1$. On the reference element $\widehat{K}$, the divergence conforming element space $\widehat{\mathbf{W}}(\widehat{K})$ is defined by the subspace of

$$
\begin{array}{r}
\left(Q_{k+1, k, k} \backslash\left\{\hat{y}^{k} \hat{z}^{k} P_{k+1}(\hat{x})\right\}\right) \times\left(Q_{k, k+1, k} \backslash\left\{\hat{x}^{k} \hat{z}^{k} P_{k+1}(\hat{y})\right\}\right) \times \\
\left(Q_{k, k, k+1} \backslash\right. \\
\\
\left.\left\{\hat{x}^{k} \hat{y}^{k} P_{k+1}(\hat{z})\right\}\right),
\end{array}
$$

where the vectors $\left\{\hat{\mathbf{a}}_{i j}\right\}_{j=1,2}$ are replaced by $\hat{\mathbf{b}}_{i}$ for $i=1,2,3$ as follows:

$$
\begin{aligned}
& \hat{\mathbf{a}}_{11}=\left\{\left(\hat{x}^{k+1} \hat{y}^{k} \hat{z}^{\ell}, 0,0\right)\right\}_{\ell=0}^{k-1} \\
& \hat{\mathbf{a}}_{12}=\left\{\left(0, \hat{x}^{k} \hat{y}^{k+1} \hat{z}^{\ell}, 0\right)\right\}_{\ell=0}^{k-1} \Rightarrow \hat{\mathbf{b}}_{1}=\left\{\left(\hat{x}^{k+1} \hat{y}^{k} \hat{z}^{\ell},-\hat{x}^{k} \hat{y}^{k+1} \hat{z}^{\ell}, 0\right)\right\}_{\ell=0}^{k-1} \text {, } \\
& \hat{\mathbf{a}}_{21}=\left\{\left(0, \hat{x}^{\ell} \hat{y}^{k+1} \hat{z}^{k}, 0\right)\right\}_{\ell=0}^{k-1} \Rightarrow \hat{\mathbf{b}}_{2}=\left\{\left(0, \hat{x}^{\ell} \hat{y}^{k+1} \hat{z}^{k},-\hat{x}^{\ell} \hat{y}^{k} \hat{z}^{k+1}\right)\right\}_{\ell=0}^{k-1} \text {, } \\
& \hat{\mathbf{a}}_{22}=\left\{\left(0,0, \hat{x}^{\ell} \hat{y}^{k} \hat{z}^{k+1}\right)\right\}_{\ell=0}^{k-1} \\
& \hat{\mathbf{a}}_{31}=\left\{\left(\hat{x}^{k+1} \hat{y}^{\ell} \hat{z}^{k}, 0,0\right)\right\}_{\ell=0}^{k-1} \Rightarrow \hat{\mathbf{b}}_{3}=\left\{\left(\hat{x}^{k+1} \hat{y}^{\ell} \hat{z}^{k}, 0,-\hat{x}^{k} \hat{y}^{\ell} \hat{z}^{k+1}\right)\right\}_{\ell=0}^{k-1} \text {. } \\
& \hat{\mathbf{a}}_{32}=\left\{\left(0,0, \hat{x}^{k} \hat{y}^{\ell} \hat{z}^{k+1}\right)\right\}_{\ell=0}^{k-1}
\end{aligned}
$$

Then we see the dimension of $\widehat{\mathbf{W}}(\widehat{K})$ is $3(k+1)^{2}(k+2)-3(k+2)-3 k$. 
To define the degrees of freedom, we need an auxiliary space. We define $\widehat{\Psi}_{k}^{\text {div }}(\widehat{K})$ to be the subspace of $\left(Q_{k-1, k, k} \backslash\left\{\hat{y}^{k} \hat{z}^{k} P_{k-1}(\hat{x})\right\}\right) \times$ $\left(Q_{k, k-1, k} \backslash\left\{\hat{x}^{k} \hat{z}^{k} P_{k-1}(\hat{y})\right\}\right) \times\left(Q_{k, k, k-1} \backslash\left\{\hat{x}^{k} \hat{y}^{k} P_{k-1}(\hat{z})\right\}\right)$, where vectors $\left\{\hat{\phi}_{i j}\right\}_{j=1}^{2}$ are replaced by $\hat{\boldsymbol{v}}_{i}$ for $i=1,2,3$ as follows:

$$
\begin{aligned}
& \hat{\phi}_{11}=\left\{\left(\hat{x}^{k-1} \hat{y}^{k} \hat{z}^{\ell}, 0,0\right)\right\}_{\ell=0}^{k-1} \\
& \hat{\phi}_{12}=\left\{\left(0, \hat{x}^{k} \hat{y}^{k-1} \hat{z}^{\ell}, 0\right)\right\}_{\ell=0}^{k-1} \\
& \Rightarrow \hat{\boldsymbol{\psi}}_{1}=\left\{\left(\hat{x}^{k-1} \hat{y}^{k} \hat{z}^{\ell},-\hat{x}^{k} \hat{y}^{k-1} \hat{z}^{\ell}, 0\right)\right\}_{\ell=0}^{k-1}, \\
& \hat{\phi}_{21}=\left\{\left(0, \hat{x}^{\ell} \hat{y}^{k-1} \hat{z}^{k}, 0\right)\right\}_{\ell=0}^{k-1} \\
& \hat{\phi}_{22}=\left\{\left(0,0, \hat{x}^{\ell} \hat{y}^{k} \hat{z}^{k-1}\right)\right\}_{\ell=0}^{k-1} \\
& \Rightarrow \hat{\boldsymbol{\psi}}_{2}=\left\{\left(0, \hat{x}^{\ell} \hat{y}^{k-1} \hat{z}^{k},-\hat{x}^{\ell} \hat{y}^{k} \hat{z}^{k-1}\right)\right\}_{\ell=0}^{k-1}, \\
& \hat{\phi}_{31}=\left\{\left(\hat{x}^{k-1} \hat{y}^{\ell} \hat{z}^{k}, 0,0\right)\right\}_{\ell=0}^{k-1} \\
& \hat{\phi}_{32}=\left\{\left(0,0, \hat{x}^{k} \hat{y}^{\ell} \hat{z}^{k-1}\right)\right\}_{\ell=0}^{k-1} \\
& \Rightarrow \hat{\boldsymbol{\psi}}_{3}=\left\{\left(\hat{x}^{k-1} \hat{y}^{\ell} \hat{z}^{k}, 0,-\hat{x}^{k} \hat{y}^{\ell} \hat{z}^{k-1}\right)\right\}_{\ell=0}^{k-1} .
\end{aligned}
$$

Note that the definition of $\widehat{\Psi}_{k}^{\text {div }}(\widehat{K})$ is similar to that of $\widehat{\mathbf{W}}(\widehat{K})$ except that the highest exponent $k+1$ is replaced by $k-1$.

Definition 10. For any $\hat{\mathbf{u}} \in \widehat{\mathbf{W}}(\widehat{K})$, the degrees of freedom are given on faces $\hat{f}$ with unit normal $\hat{\mathbf{n}}$ and in the interior of $\widehat{K}$ as follows:

$$
\begin{gathered}
\int_{\hat{f}} \hat{\mathbf{u}} \cdot \hat{\mathbf{n}} \hat{q} d \hat{A}, \quad \hat{q} \in Q_{k, k}^{\prime}(\hat{f}), \quad \text { for each face } \hat{f}, \\
\int_{\hat{K}} \hat{\mathbf{u}} \cdot \hat{\mathbf{q}} d \hat{\mathbf{x}}, \quad \hat{\mathbf{q}} \in \widehat{\Psi}_{k}^{\operatorname{div}}(\widehat{K}),
\end{gathered}
$$

where $Q_{k, k}^{\prime}(\hat{f})=Q_{k, k}^{\prime}(\hat{x}, \hat{y})$ is the subspace of $Q_{k, k}(\hat{x}, \hat{y}) \backslash\left\{\hat{x}^{k} \hat{y}^{k}\right\}$ for the face $\hat{f}$ in $\hat{x} \hat{y}$-plane etc. 
Theorem 11. A vector function $\hat{\mathbf{u}} \in \widehat{\mathbf{W}}(\widehat{K})$ is uniquely determined by the degrees of freedom (10)-(11). And the finite element space $\widehat{\mathbf{W}}(\widehat{K})$ is conforming in $H(\operatorname{div}, \Omega)$.

Proof. Since the number of conditions and the dimension of $\widehat{\mathbf{W}}(\widehat{K})$ are both $6\left\{(k+1)^{2}-1\right\}+3\left\{k(k+1)^{2}-k\right\}-3 k$, it suffices to show that if all the conditions are zero, then $\hat{\boldsymbol{u}}=0$. Since $\hat{\mathbf{u}} \cdot \hat{\mathbf{n}} \in Q_{k, k}^{\prime}(\hat{f})$, it is clear that (10) implies $\hat{\mathbf{u}} \cdot \hat{\mathbf{n}}=0$ on each face and this proves the conformity in $H$ (div) space. Also, $\hat{\mathbf{u}}=\left(\hat{u}_{1}, \hat{u}_{2}, \hat{u}_{3}\right)$ satisfies $\hat{u}_{1}=\hat{x}(1-\hat{x}) \hat{v}_{1}, \hat{u}_{2}=\hat{y}$ $(1-\hat{y}) \hat{v}_{2}$, and $\hat{u}_{3}=\hat{z}(1-\hat{z}) \hat{v}_{3}$, where $\hat{\mathbf{v}}=\left(\hat{v}_{1}, \hat{v}_{2}, \hat{v}_{3}\right) \in \widehat{\Psi}_{k}^{\operatorname{div}}$. Thus if we take $\hat{\mathbf{q}}=\hat{\mathbf{v}}$ in (11), we obtain $\hat{\mathbf{u}}=0$.

Remark 12. Our element of index $k$ lies between BDDF and RTN elements of index $k$. Although BDDF element has less degrees of freedom than our element, there is no known finite element corresponding to $H$ (curl) space in relation to de Rham diagram. Our element has $6(k+1)$ fewer degrees of freedom than the RTN element, and we can construct a new $H$ (curl) conforming element which satisfies de Rham diagram.

For a generic element $K$, we define the finite element space on $K$ as

$$
\mathbf{W}(K)=\left\{\frac{1}{\operatorname{det}\left(B_{K}\right)} B_{K} \hat{\mathbf{w}} \circ F_{K}^{-1} \mid \hat{\mathbf{w}} \in \widehat{\mathbf{W}}(\widehat{K})\right\},
$$

in the usual way. Then we have the following space:

$$
\mathbf{W}_{h}=\left\{\mathbf{w} \in H(\operatorname{div}, \Omega)|\mathbf{w}|_{K} \in \mathbf{W}(K) \text { for all } K \in \tau_{h}\right\} .
$$

Using the degrees of freedom (10)-(11) transformed on $K$, we can define the corresponding projection

$$
\omega_{K}: \mathbf{H}^{k+1}(K) \rightarrow \mathbf{W}(K),
$$


for an arbitrary element $K$. Then the global projection operator $\omega_{h}$ is defined piecewise by

$$
\omega_{h} \mathbf{v}_{K}=\omega_{K}\left(\left.\mathbf{v}\right|_{K}\right)
$$

for all $K \in \tau_{h}$.

\subsection{An $L^{2}(\Omega)$ conforming finite element spaces}

We will now define a new finite element space in $L^{2}(\Omega)$ to complete the discrete de Rham complex.

Definition 13. Let $k \geq 1$. On the reference element $\widehat{K}$, the $L^{2}(\Omega)$ conforming element $\widehat{Z}(\widehat{K})$ is defined as follows:

$$
\widehat{Z}(\widehat{K})=Q_{k, k, k}^{\dagger}(\widehat{K})
$$

where $Q_{k, k, k}^{\dagger}(\widehat{K})$ is the subspace of $Q_{k, k, k}(\widehat{K})$ except constant multiple of the term $\hat{x}^{k} \hat{y}^{k} \hat{z}^{\ell}, \hat{x}^{k} \hat{y}^{\ell} \hat{z}^{k}, \hat{x}^{\ell} \hat{y}^{k} \hat{z}^{k}$, and $\hat{x}^{k} \hat{y}^{k} \hat{z}^{k}$ for $\ell=0 \ldots k-1$.

Then we see the dimension of $\widehat{Z}(\widehat{K})$ is $(k+1)^{3}-3 k-1=k^{3}+3 k^{2}$.

Remark 14. Our new element has fewer degrees of freedom than existing $L^{2}(\Omega)$ conforming finite elements used to complete the de Rham diagram. Hence using our finite elements we can effectively calculate Maxwell's equations.

For a generic element $K$, we define the finite element space on $K$ as

$$
Z(K)=\left\{\hat{q} \circ F_{K}^{-1} \mid \hat{q} \in \widehat{Z}(\widehat{K})\right\},
$$

in the usual way. Then we have the following space:

$$
Z_{h}=\left\{q \in L^{2}(\Omega)|q|_{K} \in Z(K) \text { for all } K \in \tau_{h}\right\}
$$

and define $P_{0, h}$ to be the $L^{2}(\Omega)$ orthogonal projection into $Z_{h}$. 


\section{Analysis for the Finite Element Spaces}

In this section, we will show that the finite element spaces $U \subset H^{1}(\Omega)$, $\mathbf{V} \subset H(\operatorname{curl}, \Omega), \mathbf{W} \subset H(\operatorname{div}, \Omega)$, and $Z \subset L^{2}(\Omega)$ are connected in an intimate way by the following the discrete de Rham diagram [2, 3]:

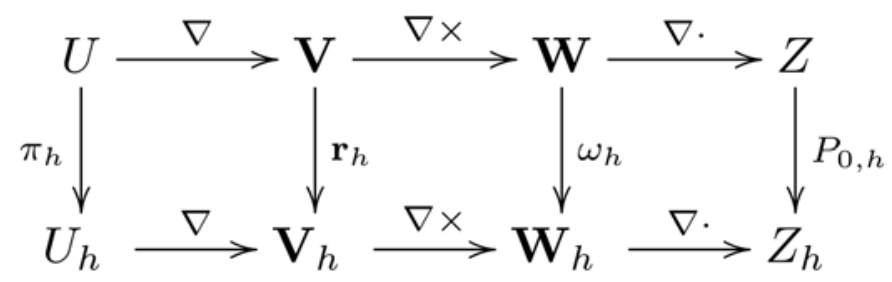

Also, we will present error estimates of the interpolation operators $\pi_{h}, \mathbf{r}_{h}, \omega_{h}$, and $P_{0, h}$.

Theorem 15. If $U_{h}$ is defined by (5) and $\mathbf{V}_{h}$ by (9), then $\nabla U_{h} \subset \mathbf{V}_{h}$. In addition, if $p$ is sufficiently smooth such that $\mathbf{r}_{h} \nabla p$ and $\pi_{h} p$ are defined, then we have $\nabla \pi_{h} p=\mathbf{r}_{h} \nabla p$.

Proof. Clearly, if $p_{h} \in U_{h}$, then we see directly that $\nabla p_{h} \in \mathbf{V}_{h}$. Hence $\nabla U_{h} \subset \mathbf{V}_{h}$.

To prove the commuting property, we map to the reference element and show that all degrees of freedom (6)-(8) vanish for $\widehat{\nabla} \pi_{\widehat{K}} \hat{p}-r_{\widehat{K}} \hat{\nabla} \hat{p}$. Then we conclude that $\widehat{\nabla} \pi_{\widehat{K}} \hat{p}-r_{\widehat{K}} \hat{\nabla} \hat{p}=0$.

For the edge degrees of freedom (6), if $\hat{\mathbf{t}}$ is tangent to $\hat{e}=[\hat{\mathbf{a}}, \hat{\mathbf{b}}]$ and $\hat{q} \in P_{k}(\hat{e})$, then using (6) and integration by parts we have

$$
\begin{aligned}
\int_{\hat{e}}\left(\hat{\nabla} \pi_{\widehat{K}} \hat{p}-\mathbf{r}_{\widehat{K}} \hat{\nabla} \hat{p}\right) \cdot \hat{\mathbf{t}} \hat{q} d \hat{s} \\
=\int_{\hat{e}}\left(\hat{\nabla} \pi_{\widehat{K}} \hat{p}-\hat{\nabla} \hat{p}\right) \cdot \hat{\mathbf{t}} \hat{q} d \hat{s}
\end{aligned}
$$




$$
\begin{aligned}
& =\int_{\hat{e}} \frac{\partial}{\partial \hat{s}}\left(\pi_{\widehat{K}} \hat{p}-\hat{p}\right) \hat{q} d \hat{s} \\
& =\left(\pi_{\widehat{K}} \hat{p}-\hat{p}\right)(\hat{\mathbf{b}})-\left(\pi_{\widehat{K}} \hat{p}-\hat{p}\right)(\hat{\mathbf{a}})-\int_{\hat{e}}\left(\pi_{\widehat{K}} \hat{p}-\hat{p}\right) \frac{\partial \hat{q}}{\partial s} d s .
\end{aligned}
$$

Since $\frac{\partial \hat{q}}{\partial \hat{s}} \in P_{k-1}(\hat{e})$ and using the vertex interpolation property and the degrees of freedom (2) for $\pi_{\widehat{K}}$, we conclude that the right-hand side above vanishes.

For the face degrees of freedom, we use the degrees of freedom in (7) together with the divergence theorem in the plane containing $\hat{f}$ to show that if $\hat{\mathbf{q}} \in \widehat{\Phi}_{k}^{\text {curl }}(\hat{f})$, then

$$
\begin{aligned}
\int_{\hat{f}}\left(\hat{\nabla} \pi_{\widehat{K}} \hat{p}-\mathbf{r}_{\widehat{K}} \hat{\nabla} \hat{p}\right) \times \hat{\mathbf{n}} \cdot \hat{\mathbf{q}} d \hat{A} \\
\quad=\int_{\hat{f}} \hat{\nabla} \hat{f}\left(\pi_{\widehat{K}} \hat{p}-\hat{p}\right) \cdot \hat{\mathbf{q}} d \hat{A} \\
\quad=\int_{\hat{\partial} f}\left(\pi_{\widehat{K}} \hat{p}-\hat{p}\right) \hat{\mathbf{n}}_{\hat{f}} \cdot \hat{\mathbf{q}} d \hat{s}-\int_{\hat{f}}\left(\pi_{\widehat{K}} \hat{p}-\hat{p}\right) \hat{\nabla} \hat{f} \cdot \hat{\mathbf{q}} d \hat{A}
\end{aligned}
$$

where $\hat{\mathbf{n}}_{\hat{f}}$ is the outward normal to $\hat{f}$. Since $\hat{\mathbf{n}}_{\hat{f}} \cdot \hat{\mathbf{q}} \in P_{k-1}(\hat{e})$ and $\hat{\nabla} \hat{f} \cdot \hat{\mathbf{q}} \in Q_{k-1, k-1}^{*}(\hat{f})$, so that right-hand side vanishes using the edge and face degrees of freedom (2) and (3) for $\pi_{\widehat{K}}$. We have thus proved that the face degrees of freedom (7) for $\widehat{\nabla} \pi_{\widehat{K}} \hat{p}$ and $\mathbf{r}_{\widehat{K}} \hat{\nabla} \hat{p}$ agree.

Finally, for the volume degrees of freedom, we use the degrees of freedom in (8) together with the integral identity to show that if $\hat{\mathbf{q}} \in \widehat{\Phi}_{k}^{\text {curl }}(\widehat{K})$, then 


$$
\begin{aligned}
\int_{\widehat{K}}\left(\hat{\nabla} \pi_{\widehat{K}} \hat{p}-\mathbf{r}_{\widehat{K}} \hat{\nabla} \hat{p}\right) \cdot \hat{\mathbf{q}} d \hat{\mathbf{x}} \\
\quad=\int_{\widehat{K}} \hat{\nabla}\left(\pi_{\widehat{K}} \hat{p}-\hat{p}\right) \cdot \hat{\mathbf{q}} d \hat{\mathbf{x}} \\
\quad=\int_{\partial \widehat{K}}\left(\pi_{\widehat{K}} \hat{p}-\hat{p}\right) \hat{\mathbf{q}} \cdot \hat{\mathbf{n}} d \hat{A}-\int_{\widehat{K}}\left(\pi_{\widehat{K}} \hat{p}-\hat{p}\right) \hat{\nabla} \cdot \hat{\mathbf{q}} d \hat{\mathbf{x}}
\end{aligned}
$$

Since $\hat{\mathbf{q}} \cdot \hat{\mathbf{n}} \in Q_{k-1, k-1}^{*}(\hat{f})$ for each face $\hat{f}$ and $\hat{\nabla} \cdot \hat{\mathbf{q}} \in Q_{k-1, k-1, k-1}^{*}(\widehat{K})$, so the right-hand side vanishes, using the face and volume degrees of freedom for $\pi_{\widehat{K}}$. This completes the proof.

Theorem 16. If $\mathbf{V}_{h}$ is defined by (9) and $\mathbf{W}_{h}$ by (12), then $\nabla \times \mathbf{V}_{h} \subset \mathbf{W}_{h}$. In addition, if $\mathbf{u}$ is smooth enough such that $\mathbf{r}_{h} \mathbf{u}$ and $\omega_{h} \nabla \times \mathbf{u}$ are defined, then $\nabla \times \mathbf{r}_{h} \mathbf{u}=\omega_{h} \nabla \times \mathbf{u}$.

Proof. The proof of $\nabla \times \mathbf{V}_{h} \subset \mathbf{W}_{h}$, see [2]. To prove the commuting property, we map to the reference element and show that the degrees of freedom given in (10) and (11) vanish for $\hat{\nabla} \times \hat{\mathbf{r}} \hat{\mathbf{u}}-\hat{\omega} \hat{\nabla} \times \hat{\mathbf{u}}$.

For the face degrees of freedom (10), we let $\hat{f}$ be a face of $\hat{K}$ with normal vector $\hat{\mathbf{n}}$ and $\hat{q} \in Q_{k, k}^{\prime}(\hat{f})$. Let $\hat{\nabla}_{\hat{f}}$ be the surface gradient. Using the definition of projection operator $\hat{\omega}$, the fact that $\left.\hat{\mathbf{n}} \cdot(\hat{\nabla} \times \hat{\mathbf{v}})\right|_{\hat{f}}=-\hat{\nabla}_{\hat{f}}$. $(\hat{\mathbf{n}} \times \hat{\mathbf{v}})$ and integration by parts, we have

$$
\begin{aligned}
\int_{\hat{f}}(\hat{\nabla} \times \hat{\mathbf{r}} \hat{\mathbf{u}}-\hat{\omega} \hat{\nabla} \times \hat{\mathbf{u}}) \cdot \hat{\mathbf{n}} \hat{q} d \hat{A} \\
=\int_{\hat{f}}(\hat{\nabla} \times \hat{\mathbf{r}} \hat{\mathbf{u}}-\hat{\nabla} \times \hat{\mathbf{u}}) \cdot \hat{\mathbf{n}} \hat{q} d \hat{A} \\
=-\int_{\hat{f}} \hat{\nabla}_{\hat{f}} \cdot(\hat{\mathbf{n}} \times(\hat{\mathbf{r}} \hat{\mathbf{u}}-\hat{\mathbf{u}})) \hat{q} d \hat{A}
\end{aligned}
$$




$$
\begin{aligned}
& =\int_{\hat{f}} \hat{\mathbf{n}} \times(\hat{\mathbf{r}} \hat{\mathbf{u}}-\hat{\mathbf{u}}) \cdot \nabla_{\hat{f}} \hat{q} d \hat{A}-\int_{\partial \hat{f}} \hat{\mathbf{n}}_{\hat{f}} \cdot(\hat{\mathbf{n}} \times(\hat{\mathbf{r}} \hat{\mathbf{u}}-\hat{\mathbf{u}})) \hat{q} d \hat{s} \\
& =\int_{\hat{f}} \hat{\mathbf{n}} \times(\hat{\mathbf{r}} \hat{\mathbf{u}}-\hat{\mathbf{u}}) \cdot \nabla_{\hat{f}} \hat{q} d \hat{A}-\int_{\partial \hat{f}}(\hat{\mathbf{r}} \hat{\mathbf{u}}-\hat{\mathbf{u}}) \cdot\left(\hat{\mathbf{n}}_{\hat{f}} \times \hat{\mathbf{n}}\right) \hat{q} d \hat{s},
\end{aligned}
$$

where $\hat{\mathbf{n}}_{\hat{f}}$ is the unit normal vector to $\hat{f}$ in the plane containing the face $\hat{f}$. Since $\hat{\nabla}_{\hat{f}} \hat{q} \in \hat{\Phi}_{k}^{\operatorname{curl}}(\hat{f})$ and $\hat{q} \in P_{k}(\hat{e})$ for each edge $\hat{e}$ of $\hat{f}$, the righthand side of above formula vanishes by the degrees of freedom (6) and (7).

For the volume degrees of freedom (11), we let $\hat{\mathbf{q}} \in \hat{\Psi}_{k}^{\operatorname{div}}(\hat{K})$. Using the definition of projection operator $\hat{\omega}$ and Green's theorem of the following form:

$$
\int_{\Omega} \nabla \times \mathbf{u} \cdot \mathbf{q} d \mathbf{x}=\int_{\Omega} \mathbf{u} \cdot \nabla \times \mathbf{q} d \mathbf{x}+\int_{\partial \Omega} \mathbf{n} \times \mathbf{u} \cdot \mathbf{q} d A
$$

we have

$$
\begin{aligned}
\int_{\hat{K}}(\hat{\nabla} \times \hat{\mathbf{r}} \hat{\mathbf{u}}-\hat{\omega} \hat{\nabla} \times \hat{\mathbf{u}}) \cdot \hat{\mathbf{q}} d \hat{\mathbf{x}} \\
\quad=\int_{\hat{K}}(\hat{\nabla} \times \hat{\mathbf{r}} \hat{\mathbf{u}}-\hat{\nabla} \times \hat{\mathbf{u}}) \cdot \hat{\mathbf{q}} d \hat{\mathbf{x}} \\
\quad=\int_{\hat{K}}(\hat{\mathbf{r}} \hat{\mathbf{u}}-\hat{\mathbf{u}}) \cdot \hat{\nabla} \times \hat{\mathbf{q}} d \hat{\mathbf{x}}+\int_{\partial \hat{K}}(\hat{\mathbf{n}} \times(\hat{\mathbf{r}} \hat{\mathbf{u}}-\hat{\mathbf{u}})) \cdot \hat{\mathbf{q}} d \hat{A}
\end{aligned}
$$

Since $\hat{\nabla} \times \hat{\mathbf{q}} \in \hat{\Psi}_{k}^{\text {curl }}(\hat{K})$ and $\hat{\mathbf{q}} \in \hat{\Phi}_{k}^{\text {curl }}(\partial \hat{K})$, the right-hand side of above formula vanishes by the degrees of freedom (7) and (8).

Now we will show that the $L^{2}(\Omega)$ projection $P_{o, h}: L^{2}(\Omega) \rightarrow Z_{h}$ is related to $\omega_{h}$.

Theorem 17. If $\mathbf{W}_{h}$, is defined by (12) and $Z_{h}$ by (13), then $\nabla \cdot \mathbf{W}_{h} \subset Z_{h}$. For all sufficiently smooth functions, we have $\nabla \cdot \omega_{h} \mathbf{u}=P_{o, h} \nabla \cdot \mathbf{u}$. 
Proof. If $\mathbf{u}_{h} \in \mathbf{W}_{h}$, then we see directly that $\nabla \cdot \mathbf{u}_{h} \in Z_{h}$.

For all $q \in Q_{k, k, k}^{\dagger}$, using the definition of $P_{o, h}$ and the Green's theorem, we obtain

$$
\begin{aligned}
& \int_{\widehat{K}}\left(\hat{\nabla} \cdot \hat{\omega} \hat{\mathbf{u}}-P_{o, h} \hat{\nabla} \cdot \hat{\mathbf{u}}\right) \hat{q} d \hat{\mathbf{V}} \\
& =\int_{\widehat{K}}(\hat{\nabla} \cdot \hat{\omega} \hat{\mathbf{u}}-\hat{\nabla} \cdot \hat{\mathbf{u}}) \hat{q} d \hat{\mathbf{V}} \\
& =\int_{\partial \widehat{K}}(\hat{\omega} \hat{\mathbf{u}}-\hat{\mathbf{u}}) \cdot \hat{\mathbf{n}} \hat{\mathbf{q}} d \hat{A}-\int_{\widehat{K}}(\hat{\omega} \hat{\mathbf{u}}-\hat{\mathbf{u}}) \cdot \hat{\nabla} \hat{q} d \hat{\mathbf{V}}
\end{aligned}
$$

Note that $q \in Q_{k, k}^{\prime}(\hat{f})$ for each face $\hat{f}$ and $\nabla q \in \widehat{\Psi}_{k}^{\operatorname{div}}(\widehat{K})$. So, both integrals on the right-hand side vanish, owing to the degrees of freedom (10) and (11).

The above result and Theorems 15 and 16 show that the discrete de Rham complex commutes. The following theorems provide error estimates for the projection operators whose proof is now standard $[1,9,10]$.

Theorem 18. Let $\tau_{h}$ be a regular family of meshes of $\Omega$. If $p \in H^{k+1}(\Omega)$ and $\mathbf{u} \in \mathbf{H}^{k+1}(\Omega)$, then there is a constant $C$ independent of $h$ such that

$$
\begin{aligned}
& \left\|p-\pi_{h} p\right\|_{1} \leq C h^{k}\|p\|_{k+1} \\
& \left\|\mathbf{u}-\mathbf{r}_{h} \mathbf{u}\right\|_{0} \leq C h^{k+1}\left(\|\mathbf{u}\|_{k+1}+\|\nabla \times \mathbf{u}\|_{k+1}\right) \\
& \left\|\mathbf{u}-\omega_{h} \mathbf{u}\right\|_{0} \leq C h^{k+1}\|\mathbf{u}\|_{k+1} \\
& \left\|p-P_{0, h} p\right\|_{0} \leq C h^{k+1}\|p\|_{k+1}
\end{aligned}
$$




\section{Acknowledgement}

This work was supported by the National Research Foundation of Korea grant funded by the Ministry of Education 2017R1C1B5017646.

\section{References}

[1] A. Alonso and A. Valli, An optimal domain decomposition preconditioner for lowfrequency time-harmonic Maxwell equations, Math. Comput. 68(226) (1999), 607-631.

DOI: https://doi.org/10.1090/S0025-5718-99-01013-3

[2] A. Bossavit, Computational Electromagnetism, Academic Press, San Diego, 1998.

[3] A. Bossavit, Mixed Finite Elements and the Complex of Whitney Forms, Academic Press, London, 1988.

[4] F. Brezzi, J. Douglas, R. Duran and M. Fortin, Mixed finite elements for second order elliptic problems in three variables, Numer. Math. 51(2) (1987), 237-250.

DOI: https://doi.org/10.1007/BF01396752

[5] J. H. Kim and Do Y. Kwak, New curl conforming finite elements on parallelepiped, Numer. Math. 131(3) (2015), 473-488.

DOI: https://doi.org/10.1007/s00211-015-0696-7

[6] J. H. Kim, New $H^{1}(\Omega)$ conforming finite elements on hexahedra, Int. J. Pure Appl. Math. 109(3) (2016), 609-618.

DOI: https://doi.org/10.12732/ijpam.v109i3.10

[7] J. C. Nedelec, Mixed finite elements in $\mathbb{R}^{3}$, Numer. Math. 35(3) (1980), 315-341.

DOI: https://doi.org/10.1007/BF01396415

[8] Philippe G. Ciarlet, The Finite Element Method for Elliptic Problems, North-Holland Publishing Company, New York, 1978.

[9] Peter Monk, Finite Element Methods for Maxwell's Equations, Clarendon Press, Oxford, 2003.

[10] Peter Monk, Analysis of a finite element method for Maxwell's equations, SIAM J. Numer. Anal. 29(3) (1992), 714-729.

DOI: https://doi.org/10.1137/0729045 